\title{
Methods for meta-analysis of pharmacodynamic dose-response data with application to multi-arm studies of alogliptin
}

Journal Title

$\mathrm{XX}(\mathrm{X}): 2-19$

(C)The Author(s) 0000

Reprints and permission:

sagepub.co.uk/journalsPermissions.nav

DOI: $10.1177 /$ ToBeAssigned

www.sagepub.com/

(S)AGE

Oliver Langford ${ }^{1}$, Jeffrey K. Aronson ${ }^{1}$, Gert van Valkenhoef ${ }^{2}$ and Richard J. Stevens ${ }^{1}$

Prepared using sagej.cls 


\begin{abstract}
Standard methods for meta-analysis of dose-response data in epidemiology assume a model with a single scalar parameter, such as log-linear relationships between exposure and outcome; such models are implicitly unbounded. In contrast, in pharmacology, multi-parameter models, such as the widely used $E_{\max }$ model, are used to describe relationships that are bounded above and below. We propose methods for estimating the parameters of a dose-response model by meta-analysis of summary data from the results of randomized controlled trials of a drug, in which each trial uses multiple doses of the drug of interest (possibly including dose 0 or placebo). We assume that, for each randomized arm of each trial, the mean and standard error of a continuous response measure and the corresponding allocated dose are available. We consider weighted least squares fitting of the model to the mean and dose pairs from all arms of all studies, and a two-stage procedure in which scalar inverse variance meta-analysis is performed at each dose, and the dose-response model is fitted to the results by weighted least squares. We then compare these with two further methods inspired by network meta-analysis that fit the model to the contrasts between doses. We illustrate the methods by estimating the parameters of the Emax model to a collection of multi-arm, multiple-dose, randomized controlled trials of alogliptin, a drug for the management of diabetes mellitus, and further examine the properties of the four methods with sensitivity analyses and a simulation study. We find that all four methods produce broadly comparable point estimates for the parameters of most interest, but a singlestage method based on contrasts between doses produces the most appropriate confidence intervals. Although simpler methods may have pragmatic advantages, such as the use of standard software for scalar meta-analysis, more sophisticated methods are nevertheless preferable for their advantages in estimation.
\end{abstract}

\title{
Keywords
}

Meta-analysis; Non-linear models; Dose response relationship, drug; Pharmacokinetics; Meta-regression; Alogliptin

\footnotetext{
${ }^{1}$ Nuffield Department of Primary Care Health Sciences, University of Oxford, ${ }^{2}$ Department of Epidemiology, University of Groningen, University Medical Center Groningen

Corresponding author:

Richard J. Stevens, Nuffield Department of Primary Care Health Sciences, Radcliffe Observatory Quarter, Woodstock Road, Oxford OX2 6GG, United Kingdom

Email: richard.stevens@phc.ox.ac.uk
}

Prepared using sagej.cls [Version: 2015/06/09 v1.01] 


\section{Introduction}

An understanding of the relationship between the drug dose or concentration and the effect that it produces is central to drug development and use. The relationship between the observed effect and the drug concentration at its site of action is known as the concentration-effect relationship; this, combined with the relationship between the administered dose and the achieved concentration, gives rise to a relationship between the administered dose and the observed effect, termed the dose-response relationship. The relationship is typically non-linear. The effect increases as the dose increases within some range of doses, but asymptotes to a maximum possible effect at high doses

12 . Knowledge of this relationship is vital to drug developers, regulatory agencies, and prescribers. However, relatively few dose-response relationships have been described in clinical studies. For example, in a survey of all issues of four pharmacology journals published between January 1980 and October 2005 (more than 18000 articles in 189 volumes), only 33 articles reported pharmacodynamic response versus time curves for four or more different doses ${ }^{3}$. Given the dearth of primary studies reporting doseresponse relationships in humans, we consider here how such relationships can be derived through secondary analyses (meta-analyses) of multiple trials.

Meta-analysis is used in many areas of medical research to collate results from individual studies, 'integrating' their findings ${ }^{4}$, and strengthening the evidence about the efficacy of a treatment ${ }^{5}$. Most meta-analysis is concerned with scalar parameters such as the $(\log )$ odds ratio, hazard ratio, or relative risk between two arms of a randomized controlled trial, or for some exposure in epidemiological studies: see for example Sattar ${ }^{6}$ and Stevens ${ }^{7}$. There is an extensive literature on meta-analysis of epidemiological studies that use a single, scalar parameter to test for the existence of "dose-response" relationships, in the sense of higher risks at higher levels of exposure. For example, Greenland ${ }^{8}$ fitted a log-linear model for the relationship between alcohol consumption and odds of cancer, of the form:

$$
\lambda(x, z)=\alpha+\beta x+\delta^{\prime} \mathbf{z}
$$

where $x$ is alcohol intake, $\mathbf{z}$ is the vector of covariates, and $\lambda$ is the log odds of being a case in the study versus being a control ${ }^{8}$. Note that this summarizes the dose-response relationship through a single scalar parameter $\beta$ and assumes no upper bound on the effect of $x$, at least within the range of doses under consideration. More recently, this method has been used to study the relationship between fruit and vegetable consumption and mortality ${ }^{9}$.

By contrast, the dose-response models used in pharmacology use multiple parameters to describe a non-linear relationship with an asymptotic maximum. A model that has found wide application as well as strong theoretical justification is the $E_{\max }$ model $^{10}$.

$$
f(\text { Dose })=E_{0}+\frac{\left(E_{\max } \times \text { Dose }\right)}{\left(E D_{50}+\text { Dose }\right)}
$$

where $f(D o s e)$ is the response, $E_{0}$ represents the null response, often measured in the presence of placebo, $\left(E_{0}+E_{\max }\right)$ is the asymptotic maximum expected response for high doses, and $E D_{50}$ is the dose that produces half of the maximum effect. Since this 
model uses multiple parameters to describe the relationship between dose and effect, it cannot be fitted by meta-analysis methods for scalar parameters.

Methods for meta-analysis of multiparameter models have previously been developed. Bivariate meta-analytic methods have been used to analyse the diagnostic accuracy of studies ${ }^{11}$, typically by fitting a bivariate normal distribution to the logittransformed sensitivity and specificity statistics. More generally, methods based on a multivariate normal assumption have been proposed for meta-analysis of studies that report multiple parameters, with potentially correlated sampling distributions, from a single dataset ${ }^{12}$. For relationships of the form (2) this approach may be feasible when multiple studies (preferably the complete set of studies in a systematic review) have published the three parameters of the $E_{\max }$ model, or when there is sufficient information from which the three parameters can be derived in each study. This approach would require, at a minimum, at least three arms of three different doses in each trial, and in addition an approximate multivariate normality assumption about the three parameters (or transformations thereof). Since the $E_{\max }$ model has strong theoretical justification and empirical support for a wide range of pharmacological systems, we hypothesized that meta-analysis methods could be developed that make explicit use of the assumption that (2) holds.

\section{Motivating Example: multi-arm studies of alogliptin}

We have analysed a data set that we have obtained from a simple systematic review of multi-arm studies of alogliptin, an oral medication for lowering blood glucose concentrations in patients with type 2 diabetes mellitus. In diabetes research glycosylated hemoglobin $\left(\mathrm{HbA}_{1 \mathrm{c}}\right)$ is a well-established biomarker for overall control of diabetes and is a suitable continuous outcome measure for dose-response analysis. We searched http://clinicaltrials.gov/ using the single search term "alogliptin" and the restriction closed (completed) trials only. We included the studies that we found in the dataset if they met the following criteria: intervention studies; daily alogliptin therapy in at least two arms of different doses; at least 12 weeks duration; the reported results included the mean change in $\mathrm{HbA}_{1 \mathrm{c}}$ from baseline to follow-up. From 32 search hits we found (Figure 1) 14 studies that met these inclusion criteria. The Appendix table shows the doses used, the numbers of participants, and the mean responses (change from baseline to follow-up) in the alogliptin and placebo arms of these 14 studies. Doses are in milligrams (mg) and dose 0 denotes a placebo arm. Figure 2 shows the $\mathrm{HbA}_{1 \mathrm{c}}$ response in each arm of each trial against the allocated dose. The data are only meant to illustrate our methods, as a number of limitations prevent the applicability of our results to clinical practice: our search was narrow and only covered ClinicalTrials.gov, the studies featured different concomitant treatments, and we later discovered that a few studies were open-label extensions of other included studies.

\section{Methods}

Estimation of the parameters of the $E_{\max }$ model is an instance of a more general problem: the estimation of a non-linear model from a collection of study reports. 
$32=$ The number of hits when searching Clin-

icalTrials.gov for multi-arm trials of alogliptin

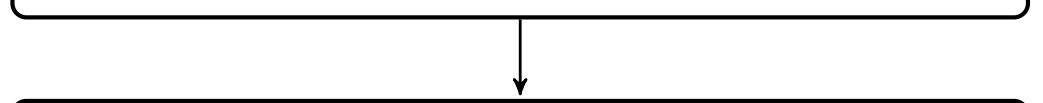

Inclusion criteria for studies: closed, report

$\mathrm{HbA}_{1 \mathrm{c}}$ results, interventional study type

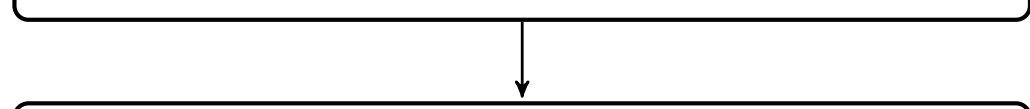

Final Results: 14 trials, split into 46

arms that spread across 6 dose categories

Figure 1. The search and inclusion criteria for alogliptin.

Suppose there are $k$ studies that have $n_{i}$ arms in each study, $i=1, \ldots, k$. Let $x_{i j}$ be some scalar characteristic of arm $j$ of study $i, 1 \leq j \leq n_{i}$, and let $y_{i j}$ be the corresponding average response. We wish to fit a non-linear model of known form:

$$
y_{i j}=\eta\left(x_{i j}, \boldsymbol{\beta}\right)+\epsilon_{i j}
$$

where $\boldsymbol{\beta}$ is a vector of $p$ unknown parameters, $\eta$ is a non-linear function describing the relationship between $x_{i j}$ and $y_{i j}$ for known $\boldsymbol{\beta}$, and $\epsilon_{i j}$ is some randomly distributed error term. For the $E_{\max }$ model, $x_{i j}$ is the allocated dose in each study arm and $\boldsymbol{\beta}=\left(E_{0}, E_{\max }, \log E D_{50}\right)^{T}$.

\subsection{Method one: arm-based inverse variance}

A naive method of analysing response data from multiple multi-arm trials, such as the data seen in Figure 2, would be to treat each arm of each study as if it were a single observation on a single subject, and proceed with standard methods for fitting equation 2. For example, a least squares estimate $\hat{\boldsymbol{\beta}}$ is the value of $\boldsymbol{\beta}$ that minimizes

$$
S=\sum_{i=1}^{k} \sum_{j=1}^{n_{i}}\left(y_{i j}-\eta\left(x_{i j}, \boldsymbol{\beta}\right)\right)^{2}
$$

This approach does not allow for the different sizes of each study or other factors that may influence the precision of estimates from different studies. For each dose category, each study that contained an arm with that dose is given equal weight. Such an unweighted approach has been used previously in meta-analyses of other non-linear models in the absence of weighted approaches ${ }^{13}$. 


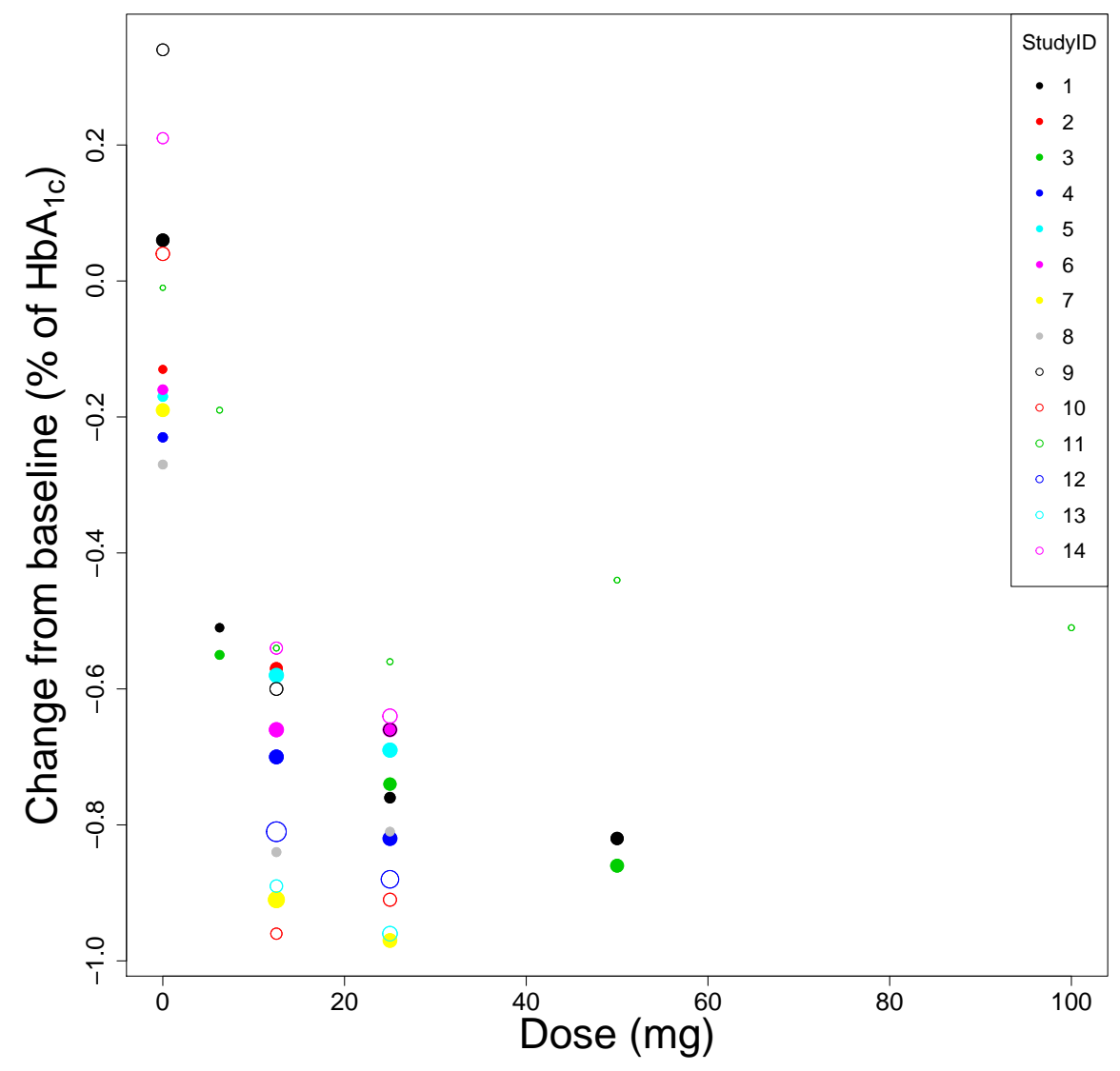

Figure 2. Changes in $\mathrm{HbA}_{1 \mathrm{c}}$ in 14 randomized trials of alogliptin, shown by allocated dose, with allocation to placebo shown as dose $0 \mathrm{mg}$, and symbol diameters inversely proportional to reported standard error.

A natural extension of this is to give weights to each data point $\left(x_{i j}, y_{i j}\right)$, defining $\hat{\boldsymbol{\beta}}$ to be the value of $\boldsymbol{\beta}$ that minimizes

$$
S=\sum_{i=1}^{k} \sum_{j=1}^{n_{i}} w_{i j}\left(y_{i j}-\eta\left(x_{i j}, \boldsymbol{\beta}\right)\right)^{2}
$$

where the weights $w_{i j}$ can take various different forms. In an obvious extension of the inverse variance method for meta-analysis ${ }^{14}$, we will use $w_{i j}=1 / s_{i j}^{2}$ where $s_{i j}$ is the estimated standard error for $y_{i j}$ reported in study $i$. Note that $\hat{\boldsymbol{\beta}}$ is then a maximum likelihood estimate (MLE) for (3) with error term $\epsilon_{i j} \sim N\left(0, s_{i j}^{2}\right)$. 


\subsection{Method two: arm-based two-stage procedure}

Method one pools information across all arms of all studies in one step. Alternatively, we could consider a two-stage procedure as follows. First, at each dose $d_{j}, 1 \leq j \leq c$, obtain an estimated effect size $\hat{y}_{j}$ and variance (standard error squared) $V_{j}$ by fixedeffects inverse variance meta-analysis (or another appropriate method for scalar metaanalysis) across all studies that have an arm at dose $d_{j}$. Secondly, having obtained estimates of the effect at each dose, obtain an estimate $\hat{\boldsymbol{\beta}}$ by fitting the model

$$
y_{j}=\eta\left(x_{j}, \boldsymbol{\beta}\right)+\epsilon_{j}
$$

to the data points $\left(d_{j}, \hat{y}_{j}\right)$. This might be done using existing software for fitting models such as (2); or by a method that incorporates the additional information in the $V_{j}$, such as minimizing

$$
S=\sum_{j=1}^{c} \frac{1}{V_{j}}\left(\hat{y}_{j}-\eta\left(d_{j}, \boldsymbol{\beta}\right)\right)^{2}
$$

Compared with Method 1, this two-stage approach might have practical advantages, making it possible to use existing software for meta-analysis in the first stage and existing or slightly modified software for dose-response algorithms in the second stage.

Another two-stage approach would be to obtain study-specific estimates $\hat{\boldsymbol{\beta}}_{\boldsymbol{i}}$ of $\boldsymbol{\beta}$ from each study with standard software, and then use methods for vector meta-analysis 12. However, this would preclude the use of any study with fewer dose arms than elements of $\beta$. In the case of model (2) this would force the exclusion of all twoarm studies, possibly the most common form of randomized controlled trial, and we therefore do not consider that approach further.

\subsection{Method three: contrast-based inverse variance}

Methods 1 and 2 use response data at the level of individual trial arms. Methods for meta-analysis of RCTs typically synthesize evidence at the level of differences or contrasts between treatment arms: because effect-modifying covariates can be expected to be approximately balanced across randomized arms, the contrasts can be expected to be much more robust than the absolute effect estimates ${ }^{14 ; 15}$. In this section and the next we consider contrast-based methods for dose-response relationships, which are possible when the following conditions are met:

$$
\eta\left(x_{i j}, \boldsymbol{\beta}\right)=\beta_{1}+\eta^{\prime}\left(x_{i j}, \boldsymbol{\beta}^{\prime}\right) ; \eta(0, \boldsymbol{\beta})=\beta_{1},
$$

for some function $\eta^{\prime}$ and where $\boldsymbol{\beta}^{\prime}=\left(\beta_{2}, \ldots, \beta_{p}\right)$. The sum (5) then becomes

$$
S=\sum_{i=1}^{k} \sum_{j=1}^{n_{i}} w_{i j}\left(y_{i j}-\beta_{1}-\eta^{\prime}\left(x_{i j}, \boldsymbol{\beta}^{\prime}\right)\right)^{2}
$$

We now generalize to the case of a study-specific parameter vector $\boldsymbol{\beta}_{i}=$ $\left(\beta_{1 i}, \beta_{2}, \ldots, \beta_{p}\right)$, in which the additive nuisance parameter $\beta_{1 i}$ varies between studies. The study-specific $\beta_{1 i}$ and the shared parameters $\boldsymbol{\beta}^{\prime}=\left(\beta_{2}, \ldots, \beta_{p}\right)$ can be estimated 
by minimizing

$$
S=\sum_{i=1}^{k} \sum_{j=1}^{n_{i}} w_{i j}\left(y_{i j}-\beta_{1 i}-\eta^{\prime}\left(x_{i j}, \boldsymbol{\beta}^{\prime}\right)\right)^{2}
$$

Because the intercept $\beta_{1 i}$ is a per-study nuisance parameter, the shared parameters $\boldsymbol{\beta}^{\prime}$ are informed by the differences in response between the arms (i.e. the contrasts). The main advantage of this per-arm formulation over pre-computing the treatment contrasts is that treatment contrasts in multi-arm trials are correlated ${ }^{16}$, which can not be accounted for in a least squares analysis.

In the particular case of the $E_{\max } \operatorname{model} \boldsymbol{\beta}_{i}=\left(E_{0 i}, E_{\max }, \log E D_{50}\right)$ and $\boldsymbol{\beta}^{\prime}=$ $\left(E_{\max }, \log E D_{50}\right)$. The parameters $E D_{50}$ and $E_{\max }$ are of the most pharmacological interest, describing the potential benefit (or harm) from the drug at various doses. The $E_{0 i}$ represent the expected outcome, in the population and setting of the $i$ th study, in the absence of treatment other than placebo.

\subsection{Method four: contrast-based estimation using network meta-analysis}

In Method 3, we introduced a study-level parameter $\beta_{1 i}, i=1, \ldots, k$, such that least squares estimation implicitly uses contrasts to estimate the remaining parameters. We now consider an explicitly contrast-based approach using network meta-analysis. As in Section 3.3 we assume the additive form (8) so that the difference between response at two doses, within trial $i$, is independent of $\beta_{1 i}$.

Network meta-analysis usually estimates contrasts between levels of a categorical exposure variable (most commonly different pharmacological substances), across a collection of studies each of which examines at least some of the contrasts ${ }^{17}$. Wandel et al., for example, studied the relative benefits of placebo, glucosamine, chondroitin, and combined glucosamine and chondroitin in patients with osteoarthritis of the hip or $\mathrm{knee}^{18}$. Network meta-analysis uses both direct and indirect evidence for each contrast, so that it is not necessary that all contrasts are present in all studies, or even that all contrasts are present in at least one study. It is sufficient that there is a connected network of contrasts in which each category is connected directly or indirectly to each other category.

In the case of dose-responsiveness, if $d$ different doses are considered across all studies in the dataset, then there are $\frac{1}{2} d(d-1)$ contrasts in total. However, they are linearly dependent, and can all be expressed in terms of the contrasts to dose 0 (allocation to placebo), $\delta_{j}=\eta^{\prime}\left(x_{j}, \boldsymbol{\beta}^{\prime}\right)$. We will use network meta-analysis to estimate the contrasts $\delta_{j}, 2 \leq j \leq d$ and the corresponding variances $V_{j}$, and then estimate $\boldsymbol{\beta}^{\prime}$ by minimizing

$$
S=\sum_{j=2}^{d} \frac{1}{V_{j}}\left(\hat{\delta}_{j}-\eta^{\prime}\left(x_{j}, \boldsymbol{\beta}^{\prime}\right)\right)^{2}
$$

An advantage of this two-stage approach is the use of existing network meta-analysis software to generate $\hat{\delta}_{j}$ and their variances. A drawback is that these estimates $\hat{\delta}_{j}$ from the network meta-analysis may be correlated, and that the two-stage approach ignores 


\begin{tabular}{r|c|c|c|c}
\hline & Method 1 & Method 2 & Method 3 & Method 4 \\
\hline$E_{0}$ & $-0.03(0.05)$ & $-0.03(0.05)$ & - & - \\
$E_{\max }$ & $-0.84(0.09)$ & $-0.84(0.08)$ & $-0.84(0.06)$ & $-0.85(0.04)$ \\
$\log E D_{50}$ & $1.05(0.62)$ & $1.05(0.57)$ & $1.23(0.36)$ & $1.31(0.23)$ \\
$I^{2}$ & $85.1 \%$ & $85.3 \% *$ & $61.6 \%$ & $64.6 \% *$ \\
\hline
\end{tabular}

Table 1. Mean (standard error) of parameter estimates and heterogeneity $I^{2}$ for each of the four methods. ${ }^{*}=I^{2}$ of the first stage (meta-analysis) only.

those correlations. An obvious variation would be to adapt existing network metaanalysis algorithms for estimation of $\delta_{j}$ to incorporate the constraints $\delta_{j}=\eta^{\prime}\left(x_{j}, \boldsymbol{\beta}^{\prime}\right)$, and hence to estimate the elements of $\boldsymbol{\beta}^{\prime}$ directly; this would be equivalent to Method 3 .

\section{Example}

We illustrate the methods of Section 3 by fitting model (2) to the dataset described in Section 2, using R software (ver. R-3.0.2, http://www.r-project.org/). Six dose categories were used across the 46 arms of the 14 studies, so that $x_{i j} \in$ $\{0,6.25,12.5,25,50,100 \mathrm{mg}\} \forall i, j$. The response variable $y_{i j}$ in $\operatorname{arm} j$ of study $i$ is the mean change in glycosylated hemoglobin $\left(\mathrm{HbA}_{1 \mathrm{c}}\right)$ from baseline to follow-up at three months, and $\sigma_{i j}$ is the within-study-arm standard deviation of the change in $\mathrm{HbA}_{1 \mathrm{c}}$. We estimate the sample standard error $s_{i j}$ from $\sigma_{i j} / \sqrt{n_{i j}}$ where $n_{i j}$ is the number of participants in arm $j$ of study $i$.

Since $E D_{50}$ is necessarily greater than 0 , while $E_{0}$ and $E_{\max }$ are supported on the entire real line, we take $\boldsymbol{\beta}=\left(E_{0}, E_{\max }, \log \left(E D_{50}\right)\right)^{T}$. Then model (2) is $E\left[Y_{i j}\right]=\eta\left(x_{i j}, \boldsymbol{\beta}\right)$ where

$$
\eta(x, \boldsymbol{\beta})=\beta_{0}+\frac{\beta_{1} x}{\exp \left(\beta_{2}\right)+x}
$$

In Table 1 columns 2, 3, and 4 show parameter estimates and standard errors from Method 1 (arm-based inverse-variance, Section 3.1). The estimated $\log \left(E D_{50}\right)$ and standard error correspond to an estimated $E D_{50}$ of $2.86 \mathrm{mg}$ with $95 \%$ confidence interval 0.85 to $9.63 \mathrm{mg}$. The estimated response under placebo, $E_{0}$, is not significantly different from 0 . The original data (grey circles) and fitted dose-response curve (black dashed line) are shown in Figure 3.

To apply Method 2 (arm-based two-stage, Section 3.2) we first conducted inversevariance meta-analysis at each dose. Not all studies included an arm with dose 0 , and only one study included dose $100 \mathrm{mg}$. The estimated responses at each dose are shown in Table 2 with standard errors, and in Figure 3 as red circles with $95 \%$ confidence intervals as vertical bars. Fitting equation (2) to these points by minimizing (7) gives the parameter estimates and standard errors shown in columns 5, 6, and 7 of Table 1. The estimated standard errors are larger than those from Method 1, and again $E_{0}$ is not significantly different from 0 . The estimated $\log \left(E D_{50}\right)$ and standard error correspond to an estimated $E D_{50}$ of $2.86 \mathrm{mg}$ with $95 \%$ confidence interval 0.94 to $8.73 \mathrm{mg}$. The corresponding dose-response curve is shown in red in Figure 3. 


\begin{tabular}{ccccc}
\hline Dose $(\mathrm{mg})$ & $\begin{array}{c}\text { Number } \\
\text { of studies }\end{array}$ & $M_{l}$ & Std. Err & $I^{2}$ \\
\hline 0.00 & 11 & -0.03 & 0.05 & 0.89 \\
6.25 & 3 & -0.48 & 0.13 & 0.71 \\
12.50 & 14 & -0.73 & 0.04 & 0.85 \\
25.00 & 14 & -0.78 & 0.04 & 0.82 \\
50.00 & 3 & -0.81 & 0.09 & 0.80 \\
100.00 & 1 & -0.51 & 0.31 & - \\
\hline
\end{tabular}

Table 2. Summary estimates from a fixed effects inverse-variance meta-analysis of the response at each dose.

\begin{tabular}{l|cc}
\hline & Estimate & Std. Error \\
\hline$\delta_{2}$ & -0.49 & 0.09 \\
$\delta_{3}$ & -0.67 & 0.04 \\
$\delta_{4}$ & -0.73 & 0.04 \\
$\delta_{5}$ & -0.81 & 0.08 \\
$\delta_{6}$ & -0.71 & 0.22 \\
\hline
\end{tabular}

Table 3. Network meta-analysis estimates of the difference in response between groups allocated to alogliptin (groups 2 to 6 ) and groups allocated to placebo (group 1).

Method 3 (contrast-based inverse-variance, Section 3.3) produced similar estimates of $E_{\max }$ (Table 1 columns 8 and 9). The estimate and standard error for $\log E D_{50}$ correspond to an estimated $E D_{50}$ of $3.42 \mathrm{mg}$ with $95 \%$ confidence interval $1.69 \mathrm{mg}$ to $6.93 \mathrm{mg}$, narrower than those from methods 1 and 2 . Note that this method does not provide an estimate of $E_{0}$; if desired, this could be obtained from meta-analysis of the placebo arms, as in the dose 0 line of Table 2.

Table 3 shows the estimated differences in responses for doses 6.25, 12.5, 25, 50, and $100 \mathrm{mg}$ compared with the reference group $(0 \mathrm{mg})$ obtained by network meta-analysis in Method 4 (Section 3.4). We then estimated $\boldsymbol{\beta}^{\prime}=\left(\log \left(E D_{50}\right), E_{\max }\right)^{T}$ from these differences and standard errors by minimizing (9). The results, shown in columns 9 and 10 of Table 1, are similar to those from Method 3, and correspond to an estimated $E D_{50}$ of $3.71 \mathrm{mg}$ with $95 \%$ confidence interval 2.36 to $5.82 \mathrm{mg}$.

Figure 4 illustrates the model fit of these four analyses using the per-arm standardized residuals, although for the two-stage methods it only shows the fit of the first (meta-analysis) stage. Comparing Method 1 (arm-based inverse-variance) to Method 3 (contrast-based inverse-variance), the overall deviance is much lower in the contrast-based model (78 versus 288). Although the contrast-based model has thirteen additional parameters for the per-study $E_{0}$, its model fit is better when judged based on the heterogeneity $I^{2}(61.6 \%$ versus $85.1 \%)$ or the Akaike Information Criterion (AIC, 110 versus 294). Method 2 (arm-based two-stage) and Method 4 (contrast-based two-stage) compare similarly. For both arm-based and contrast-based models, the fit of the one-stage model was similar to the fit of the meta-analysis stage of the two-stage models. In fact, Method 3 compares favorably to standard meta-analysis based on both 


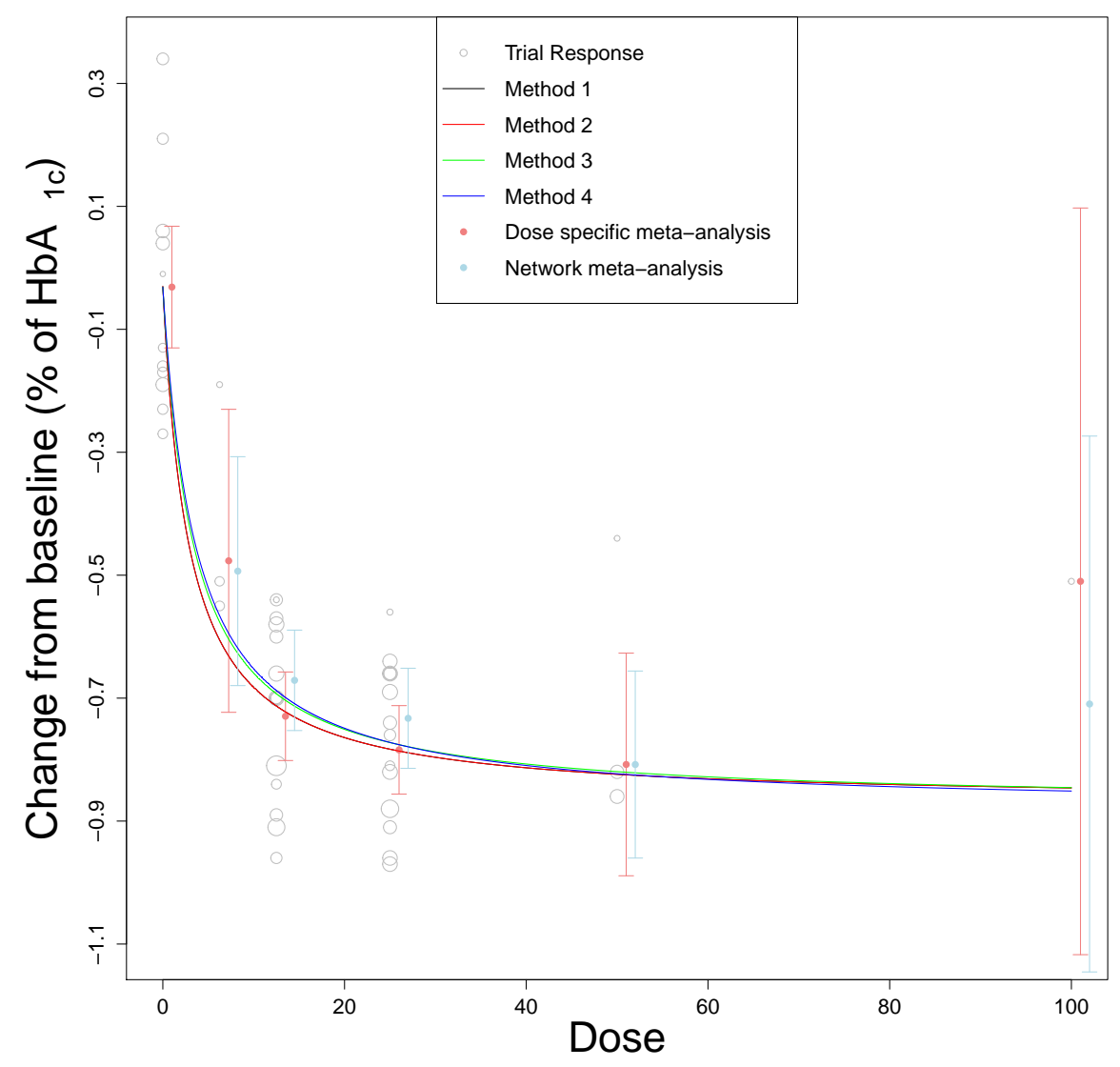

Figure 3. Fitted dose-response curves for methods 1-4. The plot displays the data, the dose-specific meta-analysis points, and the network meta-analysis estimates with $95 \%$ confidence intervals, with symbol diameters inversely proportional to standard error of estimate.

$I^{2}$ and AIC, because the dose-response model has three fewer parameters. Method 1 compares favorably to arm-based meta-analysis on $I^{2}$, but not on AIC. Thus, overall model fit and heterogeneity are improved in the contrast-based models compared with the arm-based ones, and the dose-response and standard meta-analyses fit equally well.

To examine the sensitivity of results to the exclusion of individual studies, we performed an influence analysis ${ }^{19}$, omitting one study at a time from the dataset and recalculating results. Figure 5 shows the parameter estimates and their $95 \%$ confidence intervals under each method when each study is omitted in turn. In general parameter estimates and confidence intervals, particularly for $E_{\max }$ and $E D_{50}$, are little changed by omission of any one study. The exception is that under Method 2, the confidence intervals appeared narrower with study 11 omitted. We hypothesized that this might 

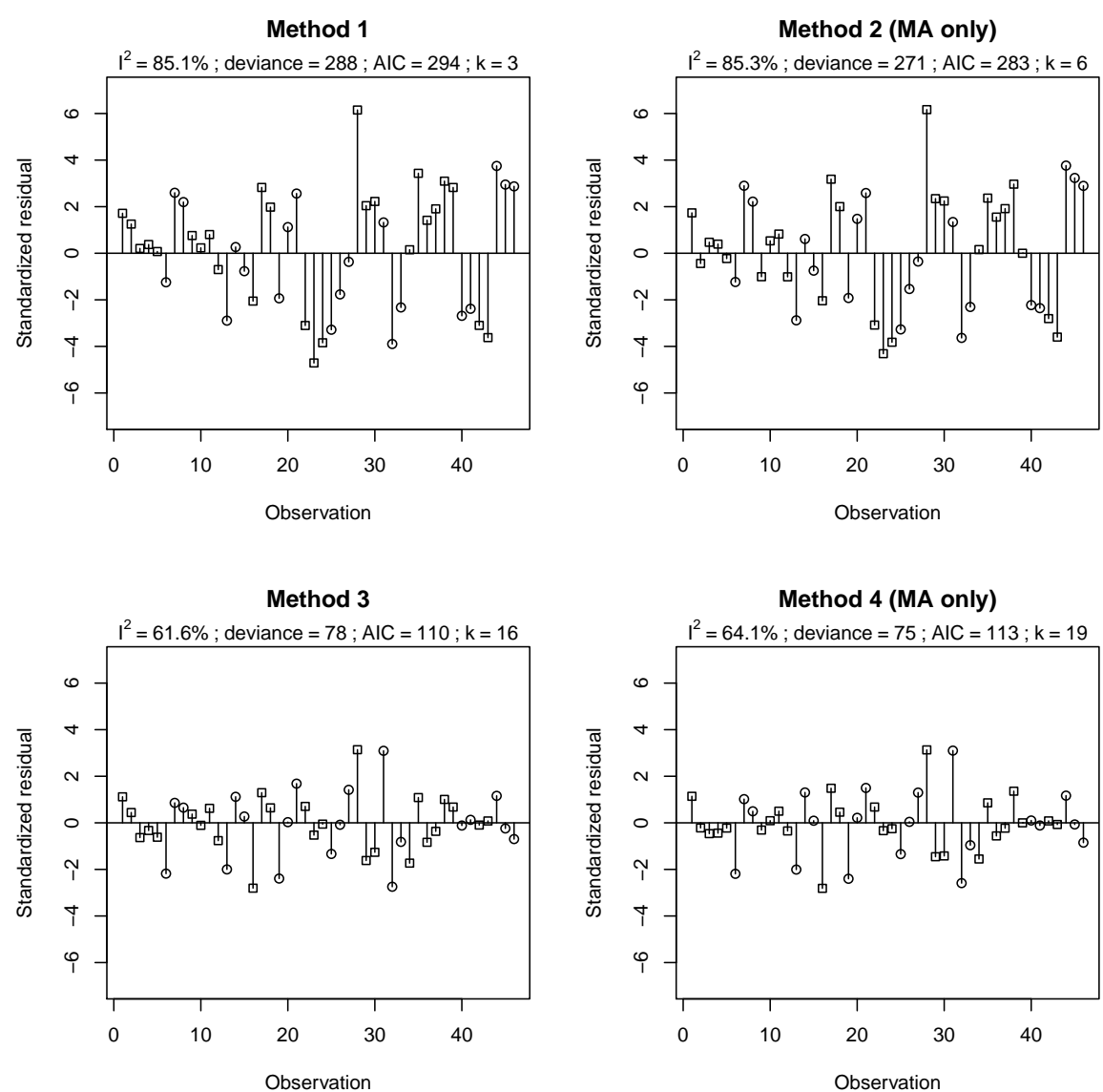

Figure 4. Per-arm standardized residuals for methods 1-4. For Methods 2 and 4 , the residuals are the residuals of the of meta-analysis only, i.e. before fitting the dose-response model. Individual studies are indicated by alternating whisker symbols (squares and circles). The heterogeneity $I^{2}$, overall deviance, Akaike Information Criterion (AIC), and the number of parameters $(k)$ are displayed on each panel.

arise because only study 11 included a dose of $100 \mathrm{mg}$ (the highest dose). We therefore conducted a sensitivity analysis, by removing the $100 \mathrm{mg}$ dose arm from study 11 and repeating both the main analysis and the influence analysis, but the sensitivity to inclusion of study 11 remained, with results almost identical to those in Figure 5 (data not shown). 


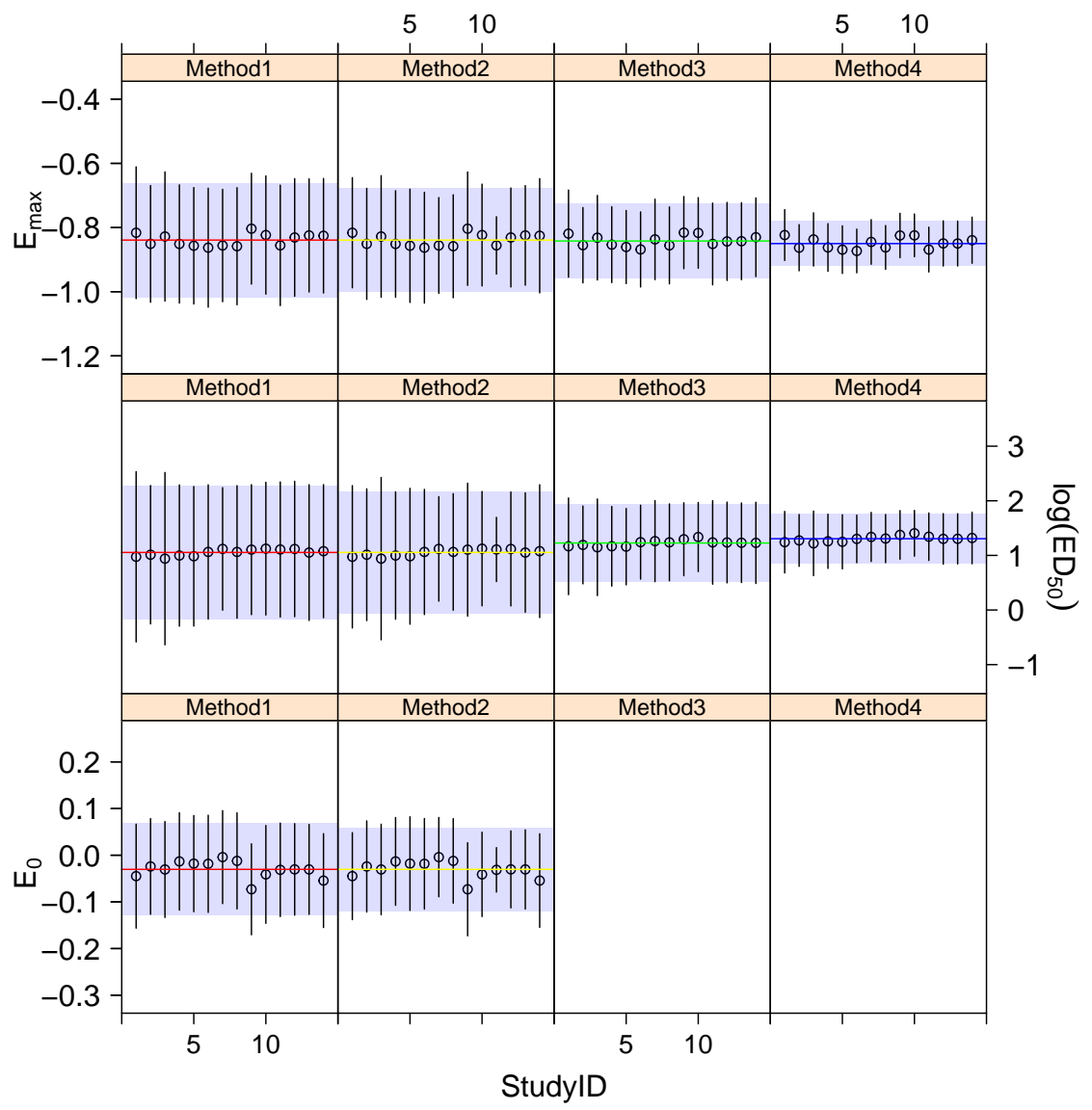

Figure 5. Sensitivity of parameter estimates and confidence intervals to the omission of individual studies. Parameter estimates (squares) and confidence intervals (vertical lines) under each method are plotted against the ID of the omitted study (x-axis). Horizontal lines and shaded areas represent the parameter estimates and $95 \%$ confidence intervals in the main analysis (see Table 1).

\section{Simulation study of performance in the presence of heterogeneity}

We conducted a simulation study to examine the properties of the methods in Section 3, including the bias in point estimates and coverage properties of confidence intervals, for the parameters of equation (2). Motivated by the alogliptin example, Section 4, individual patient data were simulated for 14 studies of between two and five arms of doses $0,6.25,12.5,25$, and $50 \mathrm{mg}$. We simulated an observed response for each patient 
$k$ of arm $j$ of study $i$, where $k=1, \ldots, n_{i j}$, as

$$
R_{i j k}=E_{0 i}+\frac{E_{\max } \times \text { Dose }_{i j k}}{E D_{50}+\text { Dose }_{i j k}}+\epsilon_{i j k}
$$

where the individual patient residuals $\epsilon_{i j k}$ were taken to be independent realizations from a normal distribution with mean 0 and standard deviation $\sigma_{i j}$.

Motivated by the estimates in Section 4 we took $E_{\max }=-0.81$ and $E D_{50}=3 \mathrm{mg}$ and took each $\sigma_{i j}$ to be the estimated residual standard error in the corresponding arm of the alogliptin dataset. We studied increasing heterogeneity of $E_{0 i}$ by simulating under four scenarios: (A) $E_{0 i}=0 \forall i$; (B) $E_{0 i} \sim N\left(0,0.05^{2}\right)$; (C) $E_{0 i} \sim N\left(0,0.1^{2}\right)$; (D) $E_{0 i} \sim N\left(0,0.2^{2}\right)$. We simulated 10,000 datasets under each scenario (1)-(4) and analysed each by the methods of Section 3.

Table 4 shows results for $E_{0}, E_{\max }$ and $E D_{50}$. In scenario $\mathrm{A}, E_{0}$ is constant across studies $\left(\sigma_{B S}=0\right)$. Column 4 shows the number of simulated samples, out of 10,000 , for which estimates were not available (NA) owing to convergence failure in the minimization algorithm, even after various initial values were used. The other columns show results across the remaining simulated data sets for which estimates were available, beginning with column 5 , which shows the Monte-Carlo error variance of the results across simulations. Columns 6 and 7 show the mean and estimated standard error (SE) of the estimates: when simulating under scenario $\mathrm{A}\left(\sigma_{B S}=0\right)$, estimates of $E_{0}$ and $E_{\max }$ appear to be unbiased, and estimates of $\log E D_{50}$ appear to underestimate slightly the true value $1.099=\log (3)$. The last column assesses the properties of the resulting $95 \%$ confidence intervals, reporting the non-coverage probability $(1-\mathrm{CP})$ : the percentage of the $95 \%$ confidence intervals that did not contain the true parameter value. In scenario A, only methods 1 and 3 had coverage probabilities close to $95 \%$.

Scenarios B, C, and D, for $\sigma_{B S}=0.05,0.10$, and 0.20 , show the corresponding results as the heterogeneity in true $E_{0 i}$ values increases. Method 1 appears to produce an unbiased estimate of the (between-studies mean) $E_{0}$ and almost unbiased estimates of $E_{\max }$ and $E D_{50}$. The standard error estimates by this method for $E_{\max }$ and $E D_{50}$ appear to be highly conservative (too large), giving nominal $95 \%$ confidence intervals with coverage much higher than $95 \%$. Method 2 consistently underestimates uncertainty, and the coverage of its confidence intervals range between $70 \%$ and $94 \%$. Method 3 results in consistent coverage close to $95 \%$ for both $E_{\max }$ and $E D_{50}$, while the two-stage variant method 4 has consistently poor coverage of $80 \%$ to $85 \%$. All methods modestly underestimate $\log E D_{50}$, corresponding to $E D_{50}$ estimates of 2.92 to $2.97 \mathrm{mg}$ when the true value is $3 \mathrm{mg}$.

\section{Discussion}

We have presented four methods for evaluating dose-response data by estimating the parameters of the $E_{\max }$ model using meta-analysis, showing the relation between the dose of a drug and its pharmacological effect. We used a dataset that described the effect of alogliptin on glycosylated haemoglobin $\left(\mathrm{HbA}_{1 \mathrm{c}}\right)$ in individuals with diabetes mellitus to illustrate the methods. The primary analysis (Section 4) and a simulation study (Section 5) showed that a single-stage model based on treatment contrasts 
Table 4. Mean, standard error (SE) and Coverage Probability (CP) of parameter estimates simulated with between-study values $\sigma_{B S} 0.00,0.05,0.10$ and 0.20.

Var denotes the Monte-Carlo (simulation) error variance. NA denotes number of simulation results, out of 10,000 , not available because of convergence failure of the algorithms in methods 1 to 4 .

\begin{tabular}{|c|c|c|c|c|c|c|c|}
\hline $\begin{array}{l}\text { Simulation } \\
\qquad\left(\sigma_{B S}\right)\end{array}$ & $\begin{array}{l}\text { Parameter } \\
\text { (True value) }\end{array}$ & Method & NA & Var & Mean & SE & $1-\mathrm{CP}$ \\
\hline \multirow[t]{3}{*}{$\begin{array}{c}\text { A. } \\
\sigma_{B S}=0\end{array}$} & $\begin{array}{c}E_{0} \\
(0.000)\end{array}$ & $\begin{array}{l}1 \\
2\end{array}$ & $\begin{array}{l}0 \\
0\end{array}$ & $\begin{array}{l}0.000 \\
0.000\end{array}$ & $\begin{array}{l}0.000 \\
0.000\end{array}$ & $\begin{array}{l}0.019 \\
0.017\end{array}$ & $\begin{array}{l}0.058 \\
0.190\end{array}$ \\
\hline & $\begin{array}{c}E_{\max } \\
(-0.810)\end{array}$ & $\begin{array}{l}1 \\
2 \\
3 \\
4\end{array}$ & $\begin{array}{c}0 \\
0 \\
0 \\
400\end{array}$ & $\begin{array}{l}0.001 \\
0.001 \\
0.001 \\
0.001\end{array}$ & $\begin{array}{l}-0.811 \\
-0.811 \\
-0.811 \\
-0.811\end{array}$ & $\begin{array}{l}0.036 \\
0.032 \\
0.036 \\
0.033\end{array}$ & $\begin{array}{l}0.057 \\
0.190 \\
0.061 \\
0.202\end{array}$ \\
\hline & $\begin{array}{c}\log E D_{50} \\
(1.099)\end{array}$ & $\begin{array}{l}1 \\
2 \\
3 \\
4\end{array}$ & $\begin{array}{c}0 \\
0 \\
0 \\
400\end{array}$ & $\begin{array}{l}0.067 \\
0.067 \\
0.068 \\
0.079\end{array}$ & $\begin{array}{l}1.079 \\
1.079 \\
1.079 \\
1.075\end{array}$ & $\begin{array}{l}0.256 \\
0.228 \\
0.257 \\
0.268\end{array}$ & $\begin{array}{l}0.047 \\
0.182 \\
0.050 \\
0.157\end{array}$ \\
\hline \multirow[t]{3}{*}{$\begin{array}{c}\text { B. } \\
\sigma_{B S}=0.05\end{array}$} & $\begin{array}{c}E_{0} \\
(0.000)\end{array}$ & $\begin{array}{l}1 \\
2\end{array}$ & $\begin{array}{l}1 \\
1\end{array}$ & $\begin{array}{l}0.001 \\
0.001\end{array}$ & $\begin{array}{l}0.000 \\
0.000\end{array}$ & $\begin{array}{l}0.025 \\
0.021\end{array}$ & $\begin{array}{l}0.053 \\
0.210\end{array}$ \\
\hline & $\begin{array}{c}E_{\max } \\
(-0.810)\end{array}$ & $\begin{array}{l}1 \\
2 \\
3 \\
4\end{array}$ & $\begin{array}{c}1 \\
1 \\
1 \\
223\end{array}$ & $\begin{array}{l}0.001 \\
0.001 \\
0.001 \\
0.001\end{array}$ & $\begin{array}{l}-0.811 \\
-0.811 \\
-0.811 \\
-0.811\end{array}$ & $\begin{array}{l}0.047 \\
0.039 \\
0.036 \\
0.032\end{array}$ & $\begin{array}{l}0.018 \\
0.153 \\
0.059 \\
0.201\end{array}$ \\
\hline & $\begin{array}{c}\log E D_{50} \\
(1.099)\end{array}$ & $\begin{array}{l}1 \\
2 \\
3 \\
4\end{array}$ & $\begin{array}{c}1 \\
1 \\
1 \\
223\end{array}$ & $\begin{array}{l}0.070 \\
0.070 \\
0.068 \\
0.079\end{array}$ & $\begin{array}{l}1.079 \\
1.079 \\
1.079 \\
1.074\end{array}$ & $\begin{array}{l}0.338 \\
0.279 \\
0.257 \\
0.268\end{array}$ & $\begin{array}{l}0.016 \\
0.142 \\
0.051 \\
0.159\end{array}$ \\
\hline \multirow[t]{3}{*}{$\begin{array}{c}\text { C. } \\
\sigma_{B S}=0.1\end{array}$} & $\begin{array}{c}E_{0} \\
(0.000)\end{array}$ & $\begin{array}{l}1 \\
2\end{array}$ & $\begin{array}{l}0 \\
0\end{array}$ & $\begin{array}{l}0.001 \\
0.001\end{array}$ & $\begin{array}{l}0.000 \\
0.000\end{array}$ & $\begin{array}{l}0.038 \\
0.029\end{array}$ & $\begin{array}{l}0.054 \\
0.246\end{array}$ \\
\hline & $\begin{array}{c}E_{\max } \\
(-0.810)\end{array}$ & $\begin{array}{l}1 \\
2 \\
3 \\
4\end{array}$ & $\begin{array}{c}0 \\
0 \\
0 \\
120\end{array}$ & $\begin{array}{l}0.002 \\
0.002 \\
0.001 \\
0.001\end{array}$ & $\begin{array}{l}-0.812 \\
-0.812 \\
-0.811 \\
-0.811\end{array}$ & $\begin{array}{l}0.071 \\
0.054 \\
0.036 \\
0.033\end{array}$ & $\begin{array}{l}0.003 \\
0.118 \\
0.060 \\
0.201\end{array}$ \\
\hline & $\begin{array}{c}\log E D_{50} \\
(1.099)\end{array}$ & $\begin{array}{l}1 \\
2 \\
3 \\
4\end{array}$ & $\begin{array}{c}0 \\
0 \\
0 \\
120\end{array}$ & $\begin{array}{l}0.077 \\
0.077 \\
0.068 \\
0.079\end{array}$ & $\begin{array}{l}1.082 \\
1.082 \\
1.079 \\
1.074\end{array}$ & $\begin{array}{l}0.510 \\
0.390 \\
0.257 \\
0.269\end{array}$ & $\begin{array}{l}0.003 \\
0.098 \\
0.050 \\
0.155\end{array}$ \\
\hline \multirow[t]{3}{*}{$\begin{array}{c}\mathrm{D} . \\
\sigma_{B S}=0.2\end{array}$} & $\begin{array}{c}E_{0} \\
(0.000)\end{array}$ & $\begin{array}{l}1 \\
2\end{array}$ & $\begin{array}{l}0 \\
0\end{array}$ & $\begin{array}{l}0.005 \\
0.005\end{array}$ & $\begin{array}{l}-0.001 \\
-0.001\end{array}$ & $\begin{array}{l}0.069 \\
0.049\end{array}$ & $\begin{array}{l}0.054 \\
0.289\end{array}$ \\
\hline & $\begin{array}{c}E_{\max } \\
(-0.810)\end{array}$ & $\begin{array}{l}1 \\
2 \\
3 \\
4\end{array}$ & $\begin{array}{c}0 \\
0 \\
0 \\
62\end{array}$ & $\begin{array}{l}0.004 \\
0.004 \\
0.001 \\
0.001\end{array}$ & $\begin{array}{l}-0.814 \\
-0.814 \\
-0.811 \\
-0.811\end{array}$ & $\begin{array}{l}0.128 \\
0.092 \\
0.036 \\
0.032\end{array}$ & $\begin{array}{c}8.0 \times 10^{-5} \\
0.100 \\
0.059 \\
0.201\end{array}$ \\
\hline & $\begin{array}{c}\log E D_{50} \\
(1.099)\end{array}$ & $\begin{array}{l}1 \\
2 \\
3 \\
4\end{array}$ & $\begin{array}{c}0 \\
0 \\
0 \\
62\end{array}$ & $\begin{array}{l}0.110 \\
0.110 \\
0.069 \\
0.080\end{array}$ & $\begin{array}{l}1.090 \\
1.090 \\
1.078 \\
1.073\end{array}$ & $\begin{array}{l}0.923 \\
0.659 \\
0.257 \\
0.268\end{array}$ & $\begin{array}{c}2.3 \times 10^{-4} \\
0.062 \\
0.050 \\
0.157\end{array}$ \\
\hline
\end{tabular}


(Section 3.3) performed best in terms of model fit and coverage probabilities. When the "placebo effect" $E_{0}$ was consistent between studies, the arm-based single-stage model (Section 3.1) performed equally well in terms of coverage, but tended to be overly conservative when there was heterogeneity in $E_{0}$. The two-stage methods (Section 3.2 and 3.4) tend to underestimate uncertainty and thus resulted in low coverage. An arguable drawback of the contrast-based methods (Section 3.3 and 3.4) is that they do not allow estimation of $E_{0}$. However, for most pharmacological applications $E_{0}$ is a nuisance parameter; if necessary it can be estimated by a separate model. The methods that are not contrast-based (Section 3.1 and 3.2) have the potentially serious drawback that they are sensitive to confounders that affect the absolute response, in addition to confounders that affect the treatment contrasts. For this reason, it is standard practice in meta-analysis to analyze treatment contrasts rather than absolute responses ${ }^{14 ; 15}$.

Determining the doses at which therapeutic benefit accrues is an important part of drug development. Knowing the dose at which the effect starts to asymptote, in this case about $20 \mathrm{mg} /$ day of alogliptin, allows one to choose the minimum dose that produces close to the maximum effect and to determine the upper limit of the dose range over which the effect can be titrated. Typically, one seeks to choose a therapeutic dosage range that encompasses between $20 \%$ and $80 \%$ of the maximum achievable effect, the range in which the dose-response curve is approximately log-linear.

Similarly, elucidation of the dose-response curves for adverse drug effects and adverse drug reactions ${ }^{2}$ can help to establish a range of doses at which benefit can be maximized and harms minimized. There is even less information about the doseresponsiveness of harms than of benefits, and a meta-analytical approach could improve our understanding of the risks of harms. In particular, a model that could assess the benefit-harm balance of a new medicine from a comparison of dose-response curves would be highly valued.

Comparisons of drugs in the same pharmacological class using these methods would allow logical therapeutic choices to be made, as in the case of the effects of bisphosphonates on bone mineral density in postmenopausal women, for which metaanalysis was performed using regression over the log-linear part of the dose-response curve $^{20}$. The investigators concluded that differences in outcomes between different bisphosphonates are a function of potency rather than pharmacological efficacy.

Drug-drug interaction studies are usually carried out at single doses of the interacting drugs. Meta-analysis of different studies in order to elucidate dose-response curves in such interactions (e.g. exploration of the dosage range over which one drug shifts the dose-response curve of another) would enhance prediction of the effects of such interactions in clinical practice.

Simultaneous pharmacokinetic-pharmacodynamic meta-analysis would result in estimates of concentration-effect curves and would aid monitoring of drug therapy using plasma concentration monitoring. This could be done using traditional compartment model-based methods or physiology-based models.

We have used the example of alogliptin to demonstrate and explore metaanalytical methods for assessing dose-responsiveness in humans. However, we have not performed a full literature search looking for studies of the effects of alogliptin, and the results should therefore be taken with caution when making inferences about effective doses of the drug. 
This dataset does not contain information regarding the effects of age, sex, race, concomitant diseases, drug-drug interactions, or any other baseline susceptibility factors ${ }^{21}$, and we have therefore not adjusted for study-level confounders that may have contributed to heterogeneity in responses. Where data allow, it would be possible to extend the model to allow for such factors if desired.

Although we have placed our results in a general, multi-parameter framework, our example and our simulation results have emphasized the three-parameter pharmacological $E_{\max }$ model that motivated this study. An obvious extension would be to the four-parameter dose-response model that includes a slope parameter known as the Hill coefficient ${ }^{22}$. This model retains the additive form necessary for methods three and four. However, in analyses that we have not reported here, the Hill coefficient in the alogliptin dataset was not significantly different from 1 at the $5 \%$ level, and therefore the $E_{\max }$ model (the sigmoid $E_{\max }$ model with Hill coefficient=1) was adequate. In principle, our methods could be applied more widely, although if applications arise in which the additivity assumption (8) is not met the two contrast-based methods would not be applicable.

The approach that we have described here is limited by the availability of data suitable for analysis. For many drugs an inadequate range of doses is used in clinical studies. Studies in which wider ranges of doses and smaller dosage increments were studied would aid analyses of this sort.

In the simulation study (section 5) we investigated between-study random variation in $E_{0}$. We have not explored how these methods perform with the introduction of random variation between studies in the coefficients $E_{\max }$ and $E D_{50}$, which is desirable for the sake of completeness. The limitations of the simulation study also include the presence of Monte-Carlo error, indicated by the variance rows of Table 4. Even after averaging over 100,000 simulated datasets this remained appreciable, and computational limits precluded the further increase of simulation sample size.

A natural extension of this work would be to fit random-effects meta-analysis models. Method 1 accounts for within-study-arm variation. However, there is value in estimating the unexplained heterogeneity that is often seen between studies and across study arms. A primary extension of this work would be to use hierarchical modelling techniques that allow a model to vary at more than one level ${ }^{23}$. A hierarchical model with a two-level structure, with study-arm estimates at level one, grouped on studies at level two, would allow the studies to have random variation about the chosen parameters. Including study-specific random effects would enable us to quantify the amount of heterogeneity between studies in the placebo effect $\left(E_{0}\right)$, the maximum effect $\left(E_{\max }\right)$, and the dose that produces $50 \%$ of the maximum $\left(E D_{50}\right)$.

To our knowledge, current dose-response methods used in systematic reviews have been based on epidemiology and air pollution studies, in which only a single parameter is estimated. Gasparrini ${ }^{24}$ used a non-linear relationship but required individual patient data (IPD) to formulate a two-stage approach, where the IPD are used in the first part to define the non-linear model. The results are then used as outcomes in the multivariate meta-analysis model. The aim is to derive a set of regression coefficients that define an average dose-response association across the studies ${ }^{24}$. In other work, related to air pollution ${ }^{25}$ the approach is to incorporate non-parametric smoothing in the metaanalysis ('meta-smoothing'). This method does not assume a particular form of model 
and lets the data determine the shape of the curve. This is appealing when looking at trend analysis, and the cited example investigated weather and seasonality in a time series ${ }^{25}$. However, pharmacological dose-response relationships require a parametric form in order to be able to determine the behaviour of the association. We have presented four methods for combining data from several multi-arm studies, and the results give an estimated form of the dose-response relationship for alogliptin where it might not have otherwise been possible.

\section{Acknowledgements}

The authors are grateful to Richard Hobbs, Jason Oke, Bethany Shinkins and the Statistics Group, in the Department of Primary Care Health Sciences at the University of Oxford, for their helpful comments and suggestions. This work was supported by the National Institutes for Health Research fellowship for Oliver Langford (grant reference MET 11-18).

\section{References}

[1] Tallarida RJ and Jacob LS. The dose-response relation in pharmacology. Springer-Verlag New York, 1979.

[2] Ferner R and Aronson J. Cato Guldberg and Peter Waage, the history of the law of mass action, and its relevance to clinical pharmacology. Br J Clin Pharmacol 2015; 0(0): 0.

[3] Hughes DA and Aronson JK. Review: A systematic review and empirical analysis of the relation between dose and duration of drug action. Journal of clinical pharmacology 2010; 50(1): 17-26. URL www. scopus. com. Cited By (since 1996):3.

[4] Glass GV. Primary, secondary, and meta-analysis of research. Educational Researcher 1976; 5(10): 3.

[5] DerSimonian R and Laird N. Meta-analysis in clinical trials. Controlled clinical trials 1986; 7(3): 177-188.

[6] Sattar N, Preiss D, Murray HM et al. Statins and risk of incident diabetes: a collaborative meta-analysis of randomised statin trials. The Lancet 2010; 375(9716): 735-742. URL www . scopus. com. Cited By (since 1996):560.

[7] Stevens RJ, Roddam AW and Beral V. Pancreatic cancer in type 1 and young-onset diabetes: Systematic review and meta-analysis. British journal of cancer 2007; 96(3): 507-509. URL www. scopus. com. Cited By (since 1996):66.

[8] Greenland S and Longnecker MP. Methods for trend estimation from summarized doseresponse data, with applications to meta-analysis. American Journal Of Epidemiology 1992; 135(11): 1301-1309.

[9] Wang X, Ouyang Y, Liu J et al. Fruit and vegetable consumption and mortality from all causes, cardiovascular disease, and cancer: Systematic review and dose-response metaanalysis of prospective cohort studies. BMJ (Online) 2014; 349.

[10] Kirby S, Brain P and Jones B. chapter Fitting Emax models to clinical trial doseresponse data. 2010.

[11] Harbord RM, Deeks JJ, Egger M et al. A unification of models for meta-analysis of diagnostic accuracy studies. Biostatistics 2007; 8(2): 239-251. URL www . scopus . com. Cited By :209.

Prepared using sagej.cls 
[12] Jackson D, Riley R and White IR. Multivariate meta-analysis: Potential and promise. Statistics in medicine 2011; 30(20): 2481-2498. URL www. scopus. com. Cited By $: 65$.

[13] Lasserson DS, Buclin T and Glasziou P. How quickly should we titrate antihypertensive medication? systematic review modelling blood pressure response from trial data. Heart 2011; 97(21): 1771-1775. Cited By (since 1996):8.

[14] Hedges LV and Olkin I. Statistical Methods for Meta-Analysis. Academic Press, 1985. ISBN 9780123363817.

[15] Egger M, Smith GD and Phillips AN. Meta-analysis: principles and procedures. BMJ 1997; 315(7121): 1533-1537. DOI:10.1136/bmj.315.7121.1533.

[16] Franchini AJ, Dias S, Ades AE et al. Accounting for correlation in network metaanalysis with multi-arm trials. Research Synthesis Methods 2012; 3(2): 142-160. DOI: 10.1002/jrsm.1049.

[17] Dias S, Sutton AJ, Ades AE et al. A generalized linear modeling framework for pairwise and network meta-analysis of randomized controlled trials. Medical Decision Making 2013; 33(5): 607-617. DOI:10.1177/0272989X12458724.

[18] Wandel S, Jni P, Tendal B et al. Effects of glucosamine, chondroitin, or placebo in patients with osteoarthritis of hip or knee: Network meta-analysis. BMJ (Online) 2010; 341(7775): 711. URL www. scopus . com. Cited By :181.

[19] Higgins JP, Green S and Collaboration C. Cochrane handbook for systematic reviews of interventions, volume 5. Wiley Online Library, 2008.

[20] Yates J. A meta-analysis characterizing the doseresponse relationships for three oral nitrogen-containing bisphosphonates in postmenopausal women. Osteoporosis International 2013; 24(1): 253-262.

[21] Aronson JK and Ferner RE. Joining the dots: new approach to classifying adverse drug reactions. BMJ: British Medical Journal 2003; 327(7425): 1222.

[22] Thomas N. Hypothesis testing and Bayesian estimation using a sigmoid Emax model applied to sparse dose-response designs. J Biopharm Stat 2006; 16(5): 657-677.

[23] Raudenbush SW and Bryk AS. Hierarchical Linear Models: Applications and Data Analysis Methods, volume 1. Sage, 2002.

[24] Gasparrini A, Armstrong B and Kenward MG. Multivariate meta-analysis for non-linear and other multi-parameter associations. Statistics in Medicine 2012; .

[25] Schwartz J and Zanobetti A. Using meta-smoothing to estimate dose-response trends across multiple studies, with application to air pollution and daily death. Epidemiology (Cambridge, Mass) 2000; 11(6): 666-672.

\section{Appendix}


Table 5. Mean response and standard deviation (SD) of HbA1c in multi-arm trials of anti-diabetic drug alogliptin in the ClinicalTrials.gov registry.

\begin{tabular}{|c|c|c|c|c|c|}
\hline Study ID & ClinicalTrialGov ID & $\begin{array}{l}\text { Dose } \\
\text { (mg) }\end{array}$ & Participants & $\begin{array}{c}\text { Response } \\
(\%)\end{array}$ & $\begin{array}{l}\mathrm{SD} \\
(\%)\end{array}$ \\
\hline \multirow[t]{5}{*}{1} & NCT01263470 & 0 & 75 & 0.06 & 0.46 \\
\hline & NCT01263470 & 6.25 & 79 & -0.51 & 0.68 \\
\hline & NCT01263470 & 12.5 & 84 & -0.7 & 0.57 \\
\hline & NCT01263470 & 25 & 79 & -0.76 & 0.55 \\
\hline & NCT01263470 & 50 & 79 & -0.82 & 0.47 \\
\hline \multirow[t]{3}{*}{2} & NCT00286455 & 0 & 63 & -0.13 & 0.63 \\
\hline & NCT00286455 & 12.5 & 131 & -0.57 & 0.63 \\
\hline & NCT00286455 & 25 & 128 & -0.66 & 0.63 \\
\hline \multirow[t]{4}{*}{3} & NCT01263496 & 6.25 & 93 & -0.55 & 0.7 \\
\hline & NCT01263496 & 12.5 & 97 & -0.7 & 0.54 \\
\hline & NCT01263496 & 25 & 94 & -0.74 & 0.52 \\
\hline & NCT01263496 & 50 & 94 & -0.86 & 0.5 \\
\hline \multirow[t]{3}{*}{4} & NCT00286494 & 0 & 95 & -0.23 & 0.67 \\
\hline & NCT00286494 & 12.5 & 196 & -0.7 & 0.67 \\
\hline & NCT00286494 & 25 & 195 & -0.82 & 0.67 \\
\hline \multirow[t]{3}{*}{5} & NCT00286468 & 0 & 97 & -0.17 & 0.67 \\
\hline & NCT00286468 & 12.5 & 201 & -0.58 & 0.67 \\
\hline & NCT00286468 & 25 & 197 & -0.69 & 0.66 \\
\hline \multirow[t]{3}{*}{6} & NCT00286442 & 0 & 103 & -0.16 & 0.68 \\
\hline & NCT00286442 & 12.5 & 210 & -0.66 & 0.68 \\
\hline & NCT00286442 & 25 & 203 & -0.66 & 0.68 \\
\hline \multirow[t]{3}{*}{7} & NCT01318070 & 0 & 115 & -0.19 & 0.55 \\
\hline & NCT01318070 & 12.5 & 111 & -0.91 & 0.44 \\
\hline & NCT01318070 & 25 & 113 & -0.97 & 0.52 \\
\hline \multirow[t]{3}{*}{8} & NCT00286429 & 0 & 126 & -0.27 & 0.82 \\
\hline & NCT00286429 & 12.5 & 130 & -0.84 & 0.82 \\
\hline & NCT00286429 & 25 & 126 & -0.81 & 0.82 \\
\hline \multirow[t]{3}{*}{9} & NCT01318083 & 0 & 103 & 0.34 & 0.61 \\
\hline & NCT01318083 & 12.5 & 104 & -0.6 & 0.56 \\
\hline & NCT01318083 & 25 & 104 & -0.66 & 0.56 \\
\hline \multirow[t]{3}{*}{10} & NCT01263483 & 0 & 74 & 0.04 & 0.46 \\
\hline & NCT01263483 & 12.5 & 76 & -0.96 & 0.55 \\
\hline & NCT01263483 & 25 & 79 & -0.91 & 0.48 \\
\hline \multirow[t]{6}{*}{11} & NCT00755846 & 0 & 41 & -0.01 & 0.86 \\
\hline & NCT00755846 & 6.25 & 42 & -0.19 & 0.78 \\
\hline & NCT00755846 & 12.5 & 42 & -0.54 & 0.79 \\
\hline & NCT00755846 & 25 & 45 & -0.56 & 0.78 \\
\hline & NCT00755846 & 50 & 43 & -0.44 & 0.81 \\
\hline & NCT00755846 & 100 & 44 & -0.51 & 0.79 \\
\hline \multirow[t]{2}{*}{12} & NCT01318122 & 12.5 & 161 & -0.81 & 0.46 \\
\hline & NCT01318122 & 25 & 163 & -0.88 & 0.52 \\
\hline \multirow[t]{2}{*}{13} & NCT01263509 & 12.5 & 106 & -0.89 & 0.59 \\
\hline & NCT01263509 & 25 & 99 & -0.96 & 0.49 \\
\hline \multirow[t]{3}{*}{14} & NCT01318109 & 0 & 100 & 0.21 & 0.64 \\
\hline & NCT01318109 & 12.5 & 92 & -0.54 & 0.56 \\
\hline & NCT01318109 & 25 & 96 & -0.64 & 0.49 \\
\hline
\end{tabular}

